\title{
Balneotherapy in Psoriasis Rehabilitation
}

\author{
IVÁN PÉTER ${ }^{1}$, ANNA JAGICZA ${ }^{1}$, ZÉNÓ AJTAY ${ }^{1}$, IMRE BONCZ ${ }^{2}$, ISTVÁN KISS ${ }^{3}$, \\ KATALIN SZENDI ${ }^{3}$, PÉTER KUSTÁN ${ }^{4}$ and BALÁZS NÉMETH ${ }^{1,3^{*}}$ \\ ${ }^{I}$ Zsigmondy Vilmos SPA Hospital, Harkany, Hungary; \\ ${ }^{2}$ Institute for Health Insurance, Faculty of Health Sciences, University of Pecs, Pecs, Hungary; \\ ${ }^{3}$ Department of Public Health Medicine, Medical School, University of Pecs, Pecs, Hungary; \\ ${ }^{4}$ Department of Laboratory Medicine, Medical School, University of Pecs, Pecs, Hungary
}

\begin{abstract}
Background/Aim: This study aimed to report a balneotherapy-based psoriasis rehabilitation protocol and assess its effectivity. Patients and Methods: Eighty psoriatic patients who underwent a 3-week-long inward balneotherapybased rehabilitation were enrolled. Psoriasis Area and Severity Index (PASI) score and high sensitivity $C$-reactive protein (CRP) were determined on admission and before discharge. Results: The mean PASI score and CRP level -determined on admission and before dischargedecreased significantly after the 3-week-long rehabilitation $7.15 \pm 7.3$ vs. $2.62 \pm 3.05(p<0.001)$ and $4.1 \pm 3.8 v s .3 .5 \pm 3.1$ $(p=0.026)$. A negative correlation was found between PASI delta and the number of spa therapies received $(r=-0.228)$. Conclusion: After completing the 3-week-long spa therapy based rehabilitation, both PASI score and CRP levels showed improvement of psoriasis. The complex spa therapy used during the rehabilitation is an effective tool to reduce the symptoms of psoriasis and improve the patient's well-being.
\end{abstract}

Psoriasis is one of the most common chronic, incurable dermatologic diseases. Its prevalence in adults is between $0.91 \%$ and $8.5 \%$ and its incidence varies from $78.9 / 100,000$ to $230 / 100,000$ (1). It is well-known that psoriasis has a considerable negative effect on quality of life due to physical and psychical symptoms (2). Furthermore, the onset of psoriasis is associated with higher cardiovascular risk, higher risk of developing diabetes, depression, inflammatory bowel disease and some malignancies $(3,4)$. The pathophysiology of

This article is freely accessible online.

Correspondence to: Dr. Balázs Németh, Department of Public Health Medicine, Medical School, University of Pécs, Szigeti St. 12. H-7624 Pécs, Hungary. Tel: +36 72536394, e-mail: balazs.nemeth@aok.pte.hu

Key Words: Psoriasis, rehabilitation, balneotherapy. psoriasis is not fully understood yet. However, several factors have been identified to play a role in the development of psoriasis. Besides genetical background and general risk factors (stress, obesity, smoking, alcohol consumption); the inordinate activation of the immune system has been identified as a key factor in the development of psoriasis (5-7).

Depending on the location and severity, psoriasis can be treated with topical agents (e.g. D-vitamin analogies, corticosteroids, dithranol), systemic medications (e.g. methotrexate, biological agents) and natural treatments (e.g. UV-light, peloid, medicinal water).

The aim of this study was to report a balneotherapy-based psoriasis rehabilitation protocol and assess its effectivity.

\section{Materials and Methods}

The present study was performed at our ISO 9001 accredited Dermatology Rehabilitation Inpatient Unit from September 2016 to May 2016. Psoriatic patients who underwent a 3-week-long inward rehabilitation were enrolled. Informed written consent was obtained from every patient. The study protocol was approved by the Regional Ethics Committee of University of Pécs, Pécs, Hungary (Permission No.: 5919/2.), in accordance with the 2008 Helsinki declaration.

Inclusion criterion was onset of psoriasis with skin lesions. Exclusion criteria were: having received any kind of balneotherapy within 1 year before admission, discontinuance of rehabilitation and withdrawal of consent.

The severity and extent of psoriasis were measured by the Psoriasis Area and Severity Index (PASI). PASI score was calculated on admission and before discharge, after completing the 3-week-long rehabilitation. To rule out interobserver error, PASI score was assessed by the same dermatologist. Besides PASI score, high sensitive $\mathrm{C}$-reactive protein (CRP) was measured from venous blood samples taken on admission and before discharge. CRP levels were determined in our university clinical laboratory with automated analyzers according to manufacturer's protocol (Cobas 8000; Roche Diagnostics GmbH, Mannheim, Germany).

Besides these objective markers of disease severity, authors aimed to find out how psoriasis affects the patient's everyday life. The involved patients were asked to fill in a $0-10$ scale 
questionnaire, which was aimed at ascertaining the patient's physical and psychical complaints (psoriasis severity, anxiety, severity of joint complaints, limitations of daily living).

The questionnaire was handed out by the dermatologist on admission and before discharge. Special attention was paid to instruct the participants that the questions need to be answered regarding their current condition. Furthermore, patients were asked to read the questions carefully, to fill in all questions and to mark only one answer to every question. To rule out potential questionnaire scaling bias, a 0-10 scale was used.

Detailed medical history and list of medications were taken from every enrolled patient.

During the 3-week-long inward rehabilitation, patients received a combination of spa treatments. The combination of treatments was designed individually to ease the symptoms of psoriasis and its complications; furthermore, comorbidities were treated as well.

Medicinal water treatment (sulphuric water) was performed in a bath tab, for $30 \mathrm{~min}, 5$ days a week. Considering the comorbidities, patients received various types of physiotherapies, electrotherapies (e.g. iontophoresis, transcutaneous electrical nerve stimulation, interference therapy), ultrasound therapies 3 times a week. UVB phototherapy, magnet therapy and transcutaneous carbon dioxide therapy were given 3 times a week in 20-min-long and 30-min long sessions. Dithranol was used 5 times a week in an ascending concentration from $0.5 \%$ to $6 \%$ according to the current dermatological status of the patient. Furthermore, sulphur (4\%), carbamide $(10 \%)$ and capsaicin containing (nonivamide - $0.01 \%$ ) ointments were used according to current guidelines of topical psoriasis treatment (8).

Statistical analysis. Statistical analysis was performed with IBM SPSS Statistics for Windows Version 22 (IBM Corp., New York, NY, USA). According to Shapiro-Wilk test, PASI scores did not show normal distribution thus, boxplot was used to demonstrate the results. Wilcoxon signed-rank test was used to compare the PASI scores and questionnaire results on admission with the PASI scores and questionnaire results before discharge. PASI delta was calculated by subtracting PASI calculated on admission from PASI calculated before discharge. Differences in PASI delta between patients receiving different kind of therapies were investigated by Mann-Witney test. To reveal correlations, the Pearson's correlation test was used. All $p$-values less than 0.05 were considered statistically significant.

\section{Results}

We enrolled 80 psoriatic patients, 35 men and 45 women. Clinical characteristics of the subjects are shown in Table I. All patients were diagnosed with psoriasis vulgaris and completed a 3-week-long inward dermatological rehabilitation. A total of 45 patients were suffering from early onset psoriasis and 35 from late onset psoriasis. Positive family history was found in $34 \%$ of the patients. Regarding the complications of psoriasis, atrophia psoriatica was present in $28 \%$, while $10 \%$ of the patients were suffering from arthritis psoriatica. Thirteen of 80 patients $(16 \%)$ were medicated with methotrexate and 5 of $80(6 \%)$ received biological therapy.
Table I. Clinical characteristics of the patients.

\begin{tabular}{lc}
\hline & Patients $\mathrm{n}=80$ \\
\hline Mean age, years & $63.7 \pm 9.1$ \\
Male, $\mathrm{n}(\%)$ & $35(44)$ \\
BMI, $\mathrm{kg} / \mathrm{m}^{2}$ & $30.2 \pm 4.5$ \\
Diabetes type $2, \mathrm{n}(\%)$ & $22(28)$ \\
Hypertension, $\mathrm{n}(\%)$ & $59(74)$ \\
Smoking, $\mathrm{n}(\%)$ & $21(26)$ \\
COPD, $\mathrm{n}(\%)$ & $7(9)$ \\
MI, $\mathrm{n}(\%)$ & $6(8)$ \\
WBC count, G/l & $6.3 \pm 3.8$ \\
Creatinine $(\mu \mathrm{mol} / \mathrm{l})$ & $81.1 \pm 15.8$ \\
eGFR (ml/min/1,73 $\left.\mathrm{m}^{2}\right)$ & $76.3 \pm 18.9$ \\
PASI on admission & $7.2 \pm 7.9$ \\
PASI before discharge & $2.6 \pm 3.3$ \\
PASI delta & $-4.9 \pm 6$ \\
CRP on admission & $4.1 \pm 3.8$ \\
CRP before discharge & $3.5 \pm 3.1$ \\
CRP delta & $-0.5 \pm 3.1$ \\
\hline
\end{tabular}

BMI: Body mass index; COPD: chronic obstructive pulmonary disease; MI: myocardial infarction; eGFR: estimated glomerular filtration rate; PASI: psoriasis area severity index.

During the 3-week-long inward rehabilitation patients received a combination of spa treatments. Every involved patient was treated with medicinal water; the detailed list of the treatments used is shown in Figure 1.

The PASI score - calculated on admission and before discharge - decreased significantly after the 3-week-long rehabilitation 7.15 vs. $2.62(p<0.001)$ as shown in Figure 2.

The CRP level - measured on admission and before discharge - decreased significantly after the 3-week-long rehabilitation $4.1 v s .3 .5(p=0.026)$ as shown in Figure 3.

All considerations evaluated by the questionnaire showed significant improvement $(p<0.001)$. Positive correlation was found between PASI score calculated on admission and disease severity $(\mathrm{r}=0.439)$, disease severity and anxiety $(\mathrm{r}=0.559)$ and disease severity and severity of joint complaints $(\mathrm{r}=0.559)$. Questionnaire results are shown in Figure 4.

A positive correlation was found between PASI delta and age $r=0.254$. A positive correlation was found between PASI calculated on admission and CRP measured on admission $(\mathrm{r}=0.396)$. A positive correlation was also found between PASI delta and CRP delta ( $r=0.389)$.

Moreover, a negative correlation was found between PASI delta and dithranol treatment $(\mathrm{r}=-0.486)$. Finally, significantly lower PASI delta scores were found in patients receiving dithranol compared to patients not receiving dithranol treatment $(p<0.001)$.

Patients received spa treatment in a combination of at least 3 ; up to 8 therapies. Negative correlation was found between PASI delta and the number of spa therapies received $(r=-0.228)$. 


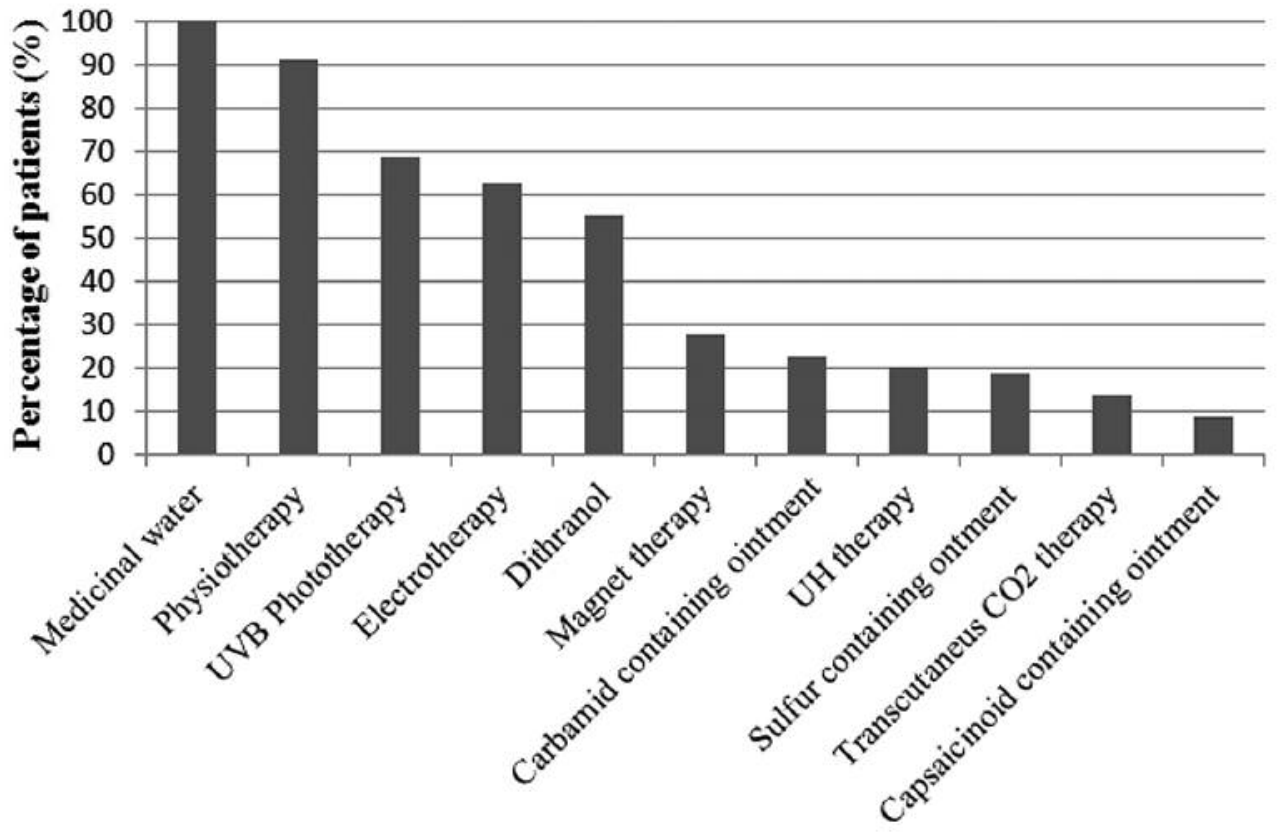

Figure 1. Detailed list of the spa therapies used.

Regarding comorbidities, PASI delta was found significantly lower among patients not suffering from hypertension $(p=0.027)$.

Comparing patients medicated with methotrexate to patients who were not treated with methotrexate, methotrexate receiving patients had significantly lower PASI scores both on admission $(p=0.015)$ and before discharge $(p=0.031)$. Being medicated with beta receptor blockers, angiotensin-converting enzyme inhibitors, angiotensin receptor blockers, calcium channel blockers, statins, oral antidiabetics, insulin, aspirin, clopidogrel, indapamide, allopurinol, diuretics, oral anticoagulants, H2-receptor blockers or proton pump inhibitors did not influence PASI scores or PASI delta.

\section{Discussion}

This study demonstrated that the PASI scores and the CRP levels decreased significantly as a result of the 3-week-long rehabilitation.

Baros et al. performed a study to compare the results of balneotherapy, standard treatment and combination treatment. The application of combined treatment showed the best short- and long-term results. However, this study did not involve inward rehabilitation and included a smaller, heterogenic population (9). To the best of our knowledge, this is the first publication which reports experiences of balneotherapy in inward psoriasis rehabilitation. The aim of the 3-week-long rehabilitation was to not only to ease the symptoms of psoriasis, but also to treat the patient's comorbidities. Taking into account that most of the patients involved were suffering from the symptoms of musculoskeletal diseases various types of physiotherapies, electrotherapies and ultrasound therapies were given to reduce the patient's musculoskeletal symptoms. Furthermore, every patient received medicinal water treatment, as it is the essential component of spa treatment. Medicinal water has been used for centuries to cure dermatological diseases particularly psoriasis. In vitro studies showed that medicinal water is capable of modulating the immune system, moreover sulfur waters can inhibit the proliferation of $\mathrm{T}$ lymphocyte, the release and production of interleukin-2 and interferon gamma (10-11). Furthermore, sulfur waters can inhibit the TNF- $\alpha$ induced expression of E-selectin and ICAM-1, which are mediators of psoriasis related inflammation (12). Exogenous hydrogen sulphide has been shown to reduce clonal growth, cell proliferation and cell adhesion of human keratinocytes in psoriasis (13). Boros et al. have found increased somatostatin release in psoriatic patients after sulfuric medicinal water treatment, which can be another possible explanation of anti-inflammatory mechanisms of sulfuric water (14). Moreover, the antiinflammatory effects of medicinal water are effective not only in psoriasis, but also in several musculoskeletal diseases (e.g. rheumatoid arthritis, fibromyalgia, ankylosing spondylitis) $(15,16)$. To enhance the beneficial effects of 


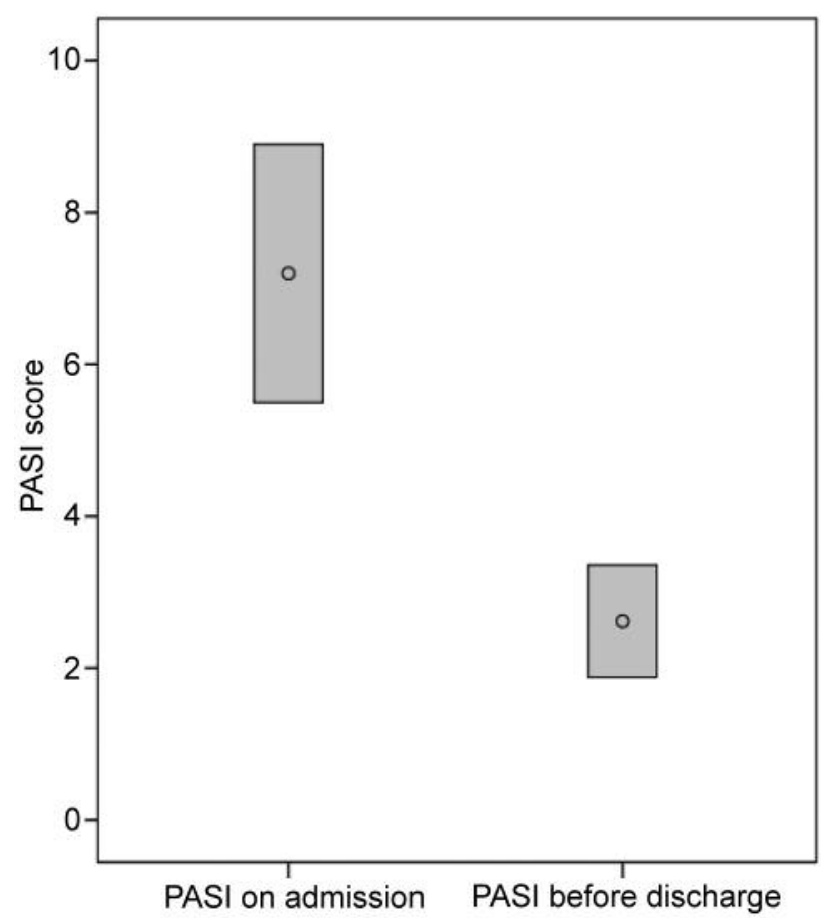

Figure 2. Changes in PASI score due to a 3-week-long rehabilitation. PASI: Psoriasis area severity index.

medicinal water on psoriasis, phototherapy (UV-B light) was used. According to previous studies, phototherapy is capable of inhibiting the proliferation of keratinocytes and suppressing the adverse $\mathrm{T}$ cell differentiation observed in psoriasis (17).

Negative correlation was found between PASI delta and dithranol treatment, which means that a greater decrease of PASI score was found among those patients who received dithranol treatment. In clinical practice dithranol is used to treat psoriatic patients with moderate or severe symptoms (18). Thus, patients receiving dithranol treatment had significantly higher PASI scores on admission compared to those who were not medicated with dithranol (10.12 vs. 3.08; $p<0.001)$. After completing the rehabilitation, PASI score decreased significantly in both patient groups, independently form dithranol treatment. In line with literature, positive correlation was found between CRP levels and PASI score on admission (19). Furthermore, a positive correlation was found between delta CRP and delta PASI, which means that the decrease in PASI score came along with the decrease in CRP levels. As CRP is a sensitive marker of inflammation, the decrease in CRP levels seen after completing the 3-weeklong rehabilitation is most likely due to the complex beneficial effects of the applied treatments. The positive correlation found between PASI scores and disease

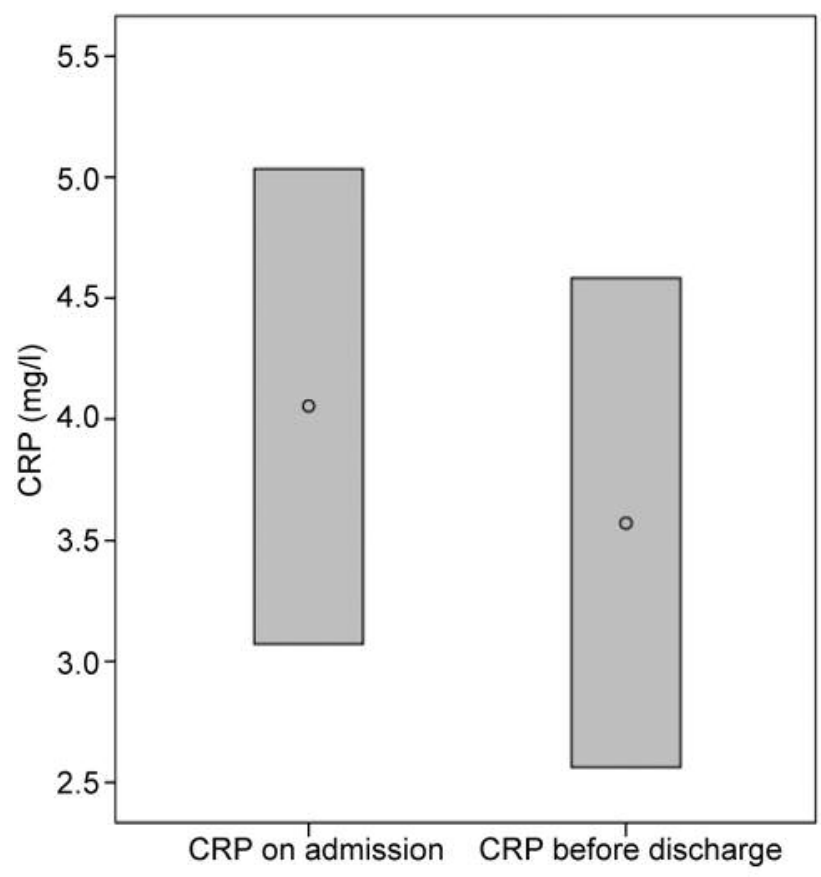

Figure 3. Changes in CRP due to 3-week long rehabilitation. CRP: C-reactive protein.

severity shown by the questionnaire indicates that patients were capable of assessing their dermatological status correctly. Not only did psoriasis severity improve after completing the 3-week-long treatment, but also, severity of joint complaints and the grade of limitation of daily living improved significantly after completing the 3-week long rehabilitation. The complex rehabilitation was capable of easing the symptoms of psoriasis and musculoskeletal diseases, which resulted in decreased anxiety and improvement in daily living. Furthermore, our results showed that the complex rehabilitation had beneficial effects on PASI score as a negative correlation was found between PASI delta and the number of spa treatments received.

In conclusion, after completing the 3-week-long spa therapy-based rehabilitation, PASI score showed improvement in psoriasis. Three-week-long rehabilitation was capable of reducing inflammation, which is indicated by the decrease in CRP levels. The complex spa therapy used during the rehabilitation is an effective tool to reduce the symptoms of psoriasis and improve the patient's well-being.

\section{Conflicts of Interest}

The Authors declare that there is no conflict of interest regarding the publication of this paper. 


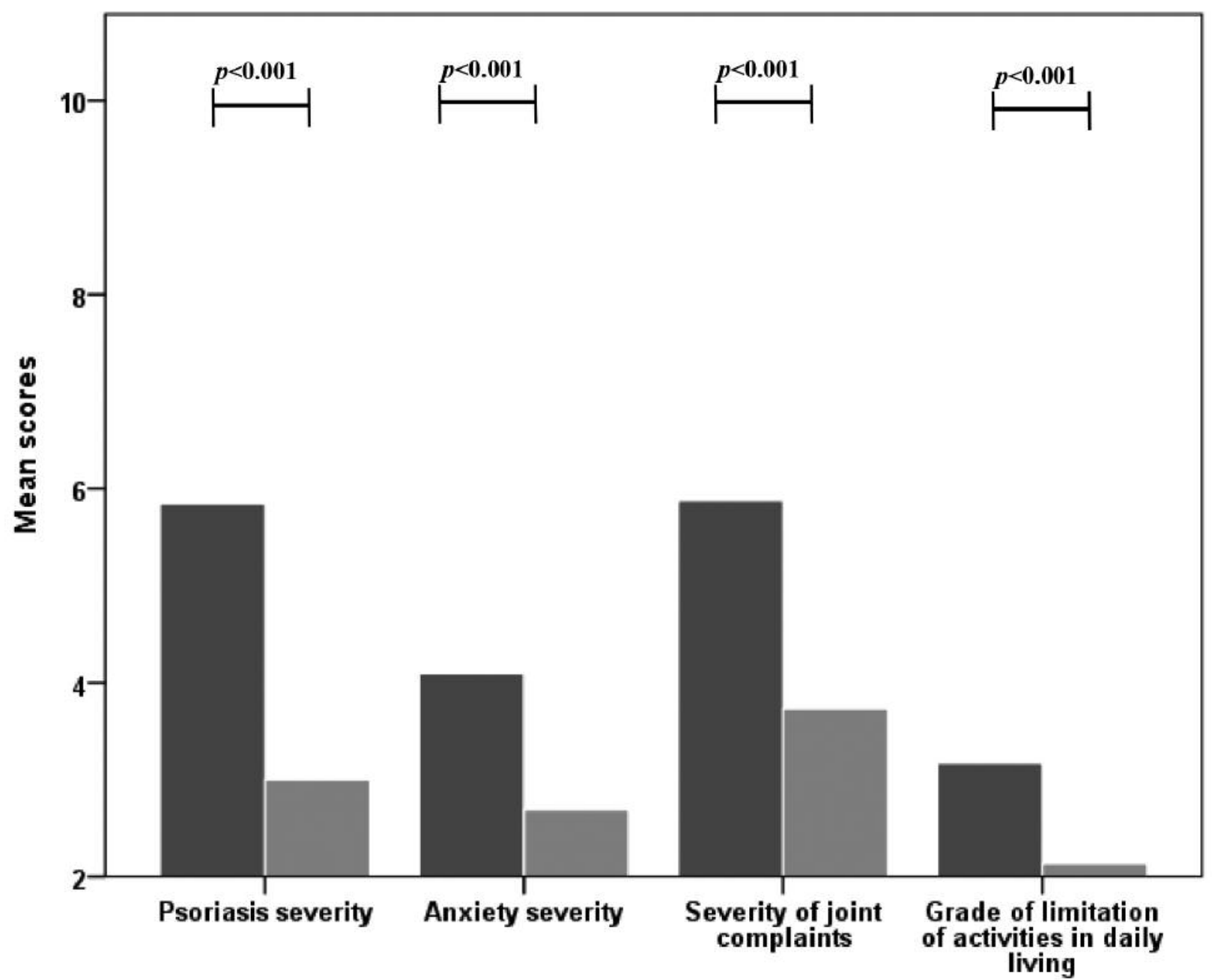

Figure 4. Questionnaire results.

\section{Acknowledgements}

The Authors would like to thank all the nurses of the Dermatology ward for their invaluable help. The present scientific contribution is dedicated to the 650th anniversary of the foundation of the University of Pécs, Hungary. Balázs Németh was supported by the ÚNKP-17-3-III New National Excellence Program of the Ministry of Human Capacities, Hungary.

\section{References}

1 Parisi R, Symmons DP, Griffiths CE, Ashcroft DM and Identification and Management of Psoriasis and Associated ComorbidiTy (IMPACT) project team: Global epidemiology of psoriasis: a systematic review of incidence and prevalence. $\mathrm{J}$ Invest Dermatol 133: 377-385, 2013.

2 Griffiths CE and Barker JN: Pathogenesis and clinical features of psoriasis. Lancet 370: 263-271, 2007.
3 Rapp SR, Feldman SR, Exum ML, Fleischer AB Jr. and Reboussin DM: Psoriasis causes as much disability as other major medical diseases. J Am Acad Dermatol 41: 401-407, 1999.

4 Smith $\mathrm{CH}$ and Barker $\mathrm{J} \mathrm{N}$ : Psoriasis and its management. BMJ 333: 380-384, 2006.

5 Huerta C, Rivero E and Garcia Rodriguez LA: Incidence and risk factors for psoriasis in the general population. Arch Dermatol 143: 1559-1565, 2007.

6 Nestle FO, Kaplan DH and Barker J Psoriasis: N Engl J Med 361: 496-509, 2009.

7 Péter I, Jagicza A, Ajtay Z, Kiss I and Németh B: Psoriasis and oxidative stress. Orv Hetil 157: 1781-1785, 2016.

8 Menter A, Korman NJ, Elmets CA, Feldman SR, Gelfand JM, Gordon KB, Gottlieb A, Koo JY, Lebwohl M, Lim HW, Van Voorhees AS, Beutner KR, Bhushan R and American Academy of Dermatology: Guidelines of care for the management of psoriasis and psoriatic arthritis. Section 3. Guidelines of care for the management and treatment of psoriasis with topical therapies. J Am Acad Dermatol 60: 643, 2009. 
9 Baros DN, Gajanin VS, Gajanin RB and Zrnić B: Comparative analysis of success of psoriasis treatment with standard therapeutic modalities and balneotherapy. Med Pregl 67: 154160, 2014.

10 Golusin Z, Jovanovic M, Jeremic B and Jolic S: Balneotherapy of Psoriasis. Serbian J Dermatol Venereol 6: 105-112, 2014.

11 Kazandjieva J, Grozdev I, Darlenski R and Tsankov N: Climatotherapy of psoriasis. Clin Dermatol 26: 477-485, 2008.

12 Castex-Rizzi N, Charveron M and Merial-Kieny C: Inhibition of TNF-alpha induced-adhesion molecules by Avene Th ermal Spring Water in human endothelial cells. J Eur Acad Dermatol Venereol 25(Suppl 1): 6-11, 2011.

13 Basavaraj KH, Darshan MS, Shanmugavelu P, Rashmi R, Mhatre AY, Dhanabal SP and Rai KS: Study on the levels of trace elements in mild and severe psoriasis. Clin Chim Acta 405: 66-70, 2009.

14 Boros M, Kemeny A, Sebok B, Bagoly T, Perkecz A, Petohazi Z, Maász G, Schmidt J, Márk L, László T, Helyes Zs, Szolcsányi J and Pintér E: Sulphurous medicinal waters increase somatostatin release: it is a possible mechanism of anti-infl ammatory effect of balneotherapy in psoriasis. Eur J Integr Med 5: 109-118, 2009.
15 Verhagen AP, Bierma-Zeinstra SM, Boers M, Cardoso JR, Lambeck J, De Bie R and De Vet HC: Balneotherapy (or spa therapy) for rheumatoid arthritis. An abridged version of Cochrane Systematic Review. Eur J Phys Rehabil Med 51: 833-847, 2015.

16 Bender T, Bálint G, Prohászka Z, Géher P and Tefner IK: Evidence-based hydro- and balneotherapy in Hungary - a systematic review and meta-analysis. Int J Biometeorol 58: 311323, 2014.

17 Racz E and Prens EP: Phototherapy and photochemotherapy for psoriasis. Dermatol Clin 33: 79-89, 2015.

18 Jekler J and Swanbeck G: One-minute dithranol therapy in psoriasis: a placebo-controlled paired comparative study. Acta Derm Venereol 72: 449-450, 1992.

19 Beygi S, Lajevardi V and Abedini R: C-reactive protein in psoriasis: a review of the literature. J Eur Acad Dermatol Venereol 28: 700-711, 2014.

Received August 3, 2017

Revised August 19, 2017

Accepted August 21, 2017 